\title{
3 Research Square \\ Clinical Analysis of Anti-NMDAR Encephalitis Combined with MOG Antibody in Children
}

Chi Hou ( $\sim$ hjtiantiankaixin@126.com )

Guangzhou Medical University https://orcid.org/0000-0002-1505-6918

Xiaojing Li

Guangzhou Women and Children's Medical Center

\section{Wenxiong Chen}

Guangzhou Women and Children's Medical Center

\section{Wenlin Wu}

Guangzhou Women and Children's Medical Center

\section{Yang Tian}

Guangzhou Women and Children's Medical Center

\section{Yani Zhang}

Guangzhou Women and Children's Medical Center

Haixia Zhu

Guangzhou Women and Children's Medical Center

\section{Yiru Zeng}

Guangzhou Women and Children's Medical Center

\section{Bingwei Peng}

Guangzhou Women and Children's Medical Center

\section{Kelu Zheng}

Guangzhou Women and Children's Medical Center

\section{Research}

Keywords: anti-NMDAR encephalitis, myelin oligodendrocyte glycoprotein antibody, children

Posted Date: November 12th, 2019

DOI: https://doi.org/10.21203/rs.2.17141/v1

License: (9) This work is licensed under a Creative Commons Attribution 4.0 International License. Read Full License 
Version of Record: A version of this preprint was published at Multiple Sclerosis and Related Disorders on July 1st, 2020. See the published version at https://doi.org/10.1016/j.msard.2020.102018. 


\section{Abstract}

Objective: To analyze the clinical features in children with anti-NMDAR encephalitis combined with myelin oligodendrocyte glycoprotein antibody (MOG ab).

Methods: Clinical data of 7 children with anti-NMDAR encephalitis combined with MOG ab were collected in Guangzhou Women and Children's Medical Center from January, 2016 to June, 2019.

Results: Average onset age was $6.0 \pm 1.3$ year-old, male to female was 2:5. Prominent symptoms in antiNMDAR encephalitis were abnormal mental behavior (7/7), sleep disorder (6/7) and speech disorder (6/7), while in MOG ab related encephalomyelitis were encephalopathy (5/7) and paralysis (4/7). 1 case developed anti-NMDAR encephalitis 1 year after recovery from MOG ab related acute disseminated encephalomyelitis (ADEM). 4 cases developed anti-NMDAR encephalitis and MOG ab related ADEM simultaneously, with 2 cases relapsed. 2 cases were anti-NMDAR encephalitis with only MOG ab positive. $10 \mathrm{MRI}$ examinations were performed in acute stage. $8 / 10$ brain MRI showed abnormalities with multiple demyelinating lesions mainly involve subcortical white matter (7/8), cortex (6/8) and basal ganglia (5/8). $2 / 10$ had long segment myelitis. Patients improved significantly after corticosteroid and intravenous immunoglobulin (IVIG) treatment. Patients with recurrent course $(n=2)$ had higher MOG ab titer (1:320) and longer duration ( $\geq 12$ months) compared with non-recurrent course $(n=5), M O G$ antibody $(\leq 1: 100)$ ( $\leq 6$ months). 3 Cases had neurological sequelae with higher cerebrospinal fluid (CSF) NMDAR antibody titer at first onset (1:320). 2 had emotional problems and 1 had epilepsy.

Conclusion: Children with anti-NMDAR encephalitis combined with MOG ab were more common in girls around 6 year-old. Patients responded well to Corticosteroid and IVIG. Cases with persistent MOG ab need longer follow-up due to recurrent risk, high titer of CSF NMDAR antibody may cause neurological sequelae. Keywords: anti-NMDAR encephalitis, myelin oligodendrocyte glycoprotein antibody, children

\section{Introduction}

Anti-N-Methyl-D-Aspartate Receptor (Anti-NMDAR) encephalitis was first reported by Dalmauet et al in $2007^{[1]}$. Myelin oligodendrocyte glycoprotein (MOG) is one of the components of myelin. Recent studies have shown that MOG antibodies can be found in different kinds of inflammatory demyelinating syndromes, and is most common in children with $A D E M^{[2]}$. Related studies have found that autoimmune encephalitis may have overlapping antibodies or combine with other autoimmune diseases such as Graves' disease and optic myelomyelitis spectrum disorder (NMOSD) ${ }^{[3,4]}$. This study reported the clinical features, treatment and prognosis of 7 children with anti-NMDAR encephalitis combined with positive MOG antibodies at Guangzhou Women and Children's Medical Center.

\section{Objects And Methods}

1.1 Study subjects: To retrospectively analyze the clinical data of 7 children with anti-NMDAR encephalitis combined with MOG antibody in Guangzhou Women and Children Medical Center from 
January, 2016 to June, 2019. Their clinical data, laboratory tests, neuroelectrophysiology, neuroimaging data, treatment and prognosis were collected.

\subsection{Methods}

1.2.1 Detection for Anti-NMDAR-IgG/IgA antibody and diagnostic criteria of anti-NMDAR encephalitis: Anti-NMDAR antibody detection kit (Euroimmun AG, Liibeek, Germany) was adopted, 30ul patient cerebrospinal fluid (as primary antibody) was incubated at room temperature for $30 \mathrm{~min}$ on a reaction slide with 4 antigens of specific expression NMDAR-transfected cells, untransfected cells (negative control), rat hippocampus and cerebellum tissue, then added goat anti-human IgG (as secondary antibody) that is labeled with fluorescein thiocyanate and incubated at room temperature for $30 \mathrm{~min}$. The slide was rinsed with phosphate buffer for $5 \mathrm{~min}$ and sealed, and the results were observed under a fluorescence microscope. The dilution ratio of the serum samples and cerebrospinal fluid was 1:10. In addition to positive cerebrospinal fluid anti-NMDAR-IgG, certain clinical features of anti-NMDAR encephalitis were required in confirming cases.

1.2.2 Detection of MOG antibody: these children's MOG-IgG in blood was detected by transfected cellsindirect immunofluorescence method: the plasmids of co-expressing human full-length MOG and GFP were transfected into human embryonic kidney 293T (HEK293T) cells. After 24h, it was fixed with 5\% paraformaldehyde for $15 \mathrm{~min}$, the patient's serum and fluorescent secondary antibody were successively incubated, and the MOG-IgG in the serum of the patient was detected by indirect immunofluorescence assay. The dilution ratio of the serum samples was 1:10.

1.2.3 Treatment: Intravenous methylprednisolone (IVMP) $(10 \mathrm{mg} / \mathrm{kg}$. $\mathrm{d}$ for 3 to 5 days, then halve the dose every 3 days to prednisone $2 \mathrm{mg} / \mathrm{kg}$. d) combined with intravenous immunoglobulin (IVIG) $(400 \mathrm{mg} / \mathrm{kg}$. d for 5 days) were performed in acute stage. Maintenance therapy continued with oral low-dose prednisone, reduced by $5 \mathrm{mg}$ every 2 weeks to $2.5 \mathrm{mg} / \mathrm{d}$ and stopped until 6 months.

1.3 Statistical analysis: Statistical analyses were conducted with SPSS version 17.0 software (SPSS Inc., Chicago IL, USA). Data were expressed as medians with ranges or mean \pm standard deviation (SD) according to the distributions.

\section{Results}

2.1 Demographic data: In this study, there were a total of 7 children with an average onset age of $6.0 \pm 1.3$ years old, male to female were 2:5. They are Han Chinese come from Guangdong, China. None of them have abnormal growth and development. 1 case had a previous history of nightmares, with frequency of 
1-2 times per day and 4-5 times per week. 1 case was diagnosed with viral encephalitis one year before onset and had a full recovery. 1 case had a history of febrile convulsion.

2.2 Clinical data: 2 cases had prodromal infection one week before onset, one was gastroenteritis, and the other was pneumonia. The manifestations of autoimmune encephalitis include: abnormal mental behavior (7/7): personality change and irritability; sleep disorder (6/7): alternating insomnia and somnolence; speech disorder (6/7): unclear pronounce and mutism; epileptic attack (4/7): 2 cases had generalized tonic-clonic seizures and the other 2 had focal seizures; involuntary movement (4/7). The manifestations of inflammatory demyelinating events include: encephalopathy (5/7), manifested by lethargy, mental fatigue, paralysis (4/7), facioplegia (3/7). Other nonspecific symptoms include hyperalgesia (3/7), headache (2/7), and fever (2/7). The modified Rankin Score (mRS) was $3.4 \pm 0.5$, and no severe patients received intensive care. Clinical data were shown in table 1.

2.3 Lab tests: A total of 10 lumbar puncture examinations were performed on 7 patients in the acute stage. CSF White blood cell (WBC) was $(35.2 \pm 25.3) \times 10^{6} / \mathrm{L}\left(10-90 \times 10^{6} / \mathrm{L}\right)$, the protein was $(0.38 \pm 0.11)$ $\mathrm{g} / \mathrm{L}(0.15-0.49 \mathrm{~g} / \mathrm{L})$, the glucose and chloride were normal. 1 case had a positive blood MOG antibody and a negative NMDAR antibody at the first onset. 1-year later, anti-NMDAR encephalitis occurred and the serum and CSF were positive for NMDAR antibody with persistent MOG antibody. In the other 6 cases, serum, CSF anti-NMDAR antibody and serum MOG antibody were simultaneously positive. The titer of blood MOG antibody was 1:100 (1:32-1:320), and the titer of CSF NMDAR antibody was 1:100 (1:101:320). Patients with recurrent course $(n=2)$ had a higher titer of MOG antibody (1:320) and a longer duration ( $\geq 6$ months), compared with non-recurrent course $(n=5), M O G$ antibody $(\leq 1: 100)$, duration(3-5 months). No children were positive for Aquaporin 4 (AQP4) antibody. 4 cases combined with other autoantibodies positive, including anti-thyroglobulin antibody and anti-thyroid peroxidase antibody (4/7), antinuclear antibodies (2/7) and anti sjogren's syndrome antibody (1/7). No associated autoimmune diseases were found. Electroencephalogram (EEG) was performed in all 7 cases in the acute phase, showing non-specific slow wave, focal slow wave (2/7), diffuse slow wave (5/7), and 2 cases complicated with epileptic discharge. See table 2.

2.4 Changes in brain MRI: A total of 10 central nervous system (CNS) MRI were performed on 7 cases in acute phase. Brain MRI abnormalities (8/10) include: subcortical white matter (7/10), cortical (6/10), basal ganglia $(5 / 10)$, thalamus $(2 / 10)$, brainstem $(2 / 10)$, cerebellum $(1 / 10)$. The lesion showed large patched long T1 and long T2 signals, with some lesions (2/10) had gadolinium enhancement. Diffuse leptomeninges enhancement was observed on brain MRI in 1 case who developed anti-NMDAR encephalitis 1 year after recovery of ADEM. 2 cases had long segment myelitis mainly involves the cervical spinal cord. There were no abnormalities in CNS MRI in 2 cases. See table 2. 
2.5 Disease course: 1 case developed anti-NMDAR encephalitis 1 year after recovery of acute disseminated encephalomyelitis (ADEM) associated with MOG antibody. 2 cases were typical antiNMDAR encephalitis accompanied by serum MOG antibody positive, without demyelinating events. These 2 case had low MOG antibody titer (1:32) and turn to negative in 3 months. 4 cases were antiNMDAR encephalitis accompanied by MOG antibody associated ADEM, 2 cases were monophasic course, another 2 cases relapsed after withdrawal of corticosteroid therapy at 6 months and had 1 relapse respectively. One patient had a recurrence of ADEM phenotype after 6 months and was finally diagnosed with NMDAR encephalitis with MOG antibody associated multiphasic disseminated encephalomyelitis (MDEM). Another case had a recurrent phenotype including anti-NMDAR encephalitis and ADEM, and recurrent NMDAR encephalitis with MOG antibody associated MDEM was finally diagnosed., These 2 children have higher MOG antibody titer(1:320) and longer duration(>1 year) compared with those of monophasic disease course $(\leq 1: 100)$ (3-6 months) at first onset. But there is no significant difference in onset age, WBC and protein in CSF and EEG change. See table 1.

2.6 Treatment and prognosis follow-up: All the 7 patients underwent first-line therapy, and the duration from onset to accept treatment was (11.1 \pm 5.4$)$ days (6-25 days) and had a good response. The 2 relapsed cases were still effective to first-line therapy and had no relapses after prolonged corticosteroid maintenance therapy for 8-month. 1 case had secondary epilepsy and had a good control after levetiracetam treatment. The 7 patients were followed up for $14.0 \pm 5.3$ months (4-21 months) from the first onset. At the last follow-up, MRI lesions were completely absorbed in 5 cases, and were mostly absorbed in 2 cases. Serum and CSF NMDAR antibody turn negative in 6 case and serum MOG antibody turn negative in 4 cases. 4 cases have full recovery, while 3 cases have neurological sequelae. Among them, 2 have emotional problems, manifested as irritability and 1 have symptomatic epilepsy. They had higher titer of CSF NMDAR antibody at the first onset (1:320) than those had full recovery $(\leq 1: 100)$.

\section{Discussion}

Anti-NMDA receptor encephalitis is the most common autoimmune encephalitis in school age children around 9 years old in which autoantibodies bind to NMDA receptors in the brain to cause inflammatory diseases of the limbic system ${ }^{[1,5,6]}$. Myelin oligodendrocyte glycoprotein (MOG) is a glycoprotein that is located on the surface of the myelin sheath and is found only in the central nervous system (CNS).The MOG antibody-related disease is an inflammatory demyelinating syndrome of CNS, manifesting monophasic or recurrent neurological dysfunction. It is more common in AQP4 IgG negative ADEM in children around 6 year-old ${ }^{[2]}$. Both MOG and anti-NMDAR antibodies can cause autoimmune encephalitis and demyelinating lesions but are different in clinical manifestations: MOG antibody-related ADEM is mainly characterized by encephalopathy, convulsions and dyskinesia with white matter patchy demyelinating lesions in brain MRI, while anti-NMDAR encephalitis, on the other hand, is characterized by 
abnormal mental behavior, often accompanied by speech and sleep disorders and other symptoms, with no significant changes in brain MRI. In clinical cases, typical cases of NMDAR antibody-positive encephalitis are easily differentiated from MOG antibody related demyelinating diseases. When clinical manifestations overlap for the two, it is difficult to identify especially with brainstem involved ${ }^{[6-8]}$.In this study, children with NMDAR encephalitis combined with MOG antibody positive were mainly girls around 6 year-old, the onset age was smaller than that of NMDAR encephalitis alone, but similar to MOG antibody related ADEM in children. Girls were more common in this study compared to NMDAR encephalitis or MOG antibodies related disease alone in children ${ }^{[6,7]}$.

In recent years, it has been found that NMDAR encephalitis can be combined with some other manifestations which suggest demyelinating episodes, including AQP4 antibody-positive NMOSD and MOG antibody-related demyelinating disease ${ }^{[4,7-9]}$. Titulaer et al firstly screened MOG antibodies for 691 patients diagnosed with NMDAR antibody encephalitis. The results showed that $1.3 \%$ of patients had anti-NMDAR encephalitis combined with MOG antibody-related demyelinating diseases successively. Most of them were female and they all had abnormalities in CNS MRI, but their clinical phenotype was less severe than that of non-demyelinating patients with anti-NMDAR encephalitis. The onset age in children is 4 to 17 years old, acute disseminated encephalomyelitis is the most common form of demyelinating phenotype. Other phenotypes include brainstem encephalitis and optic neuromyelitis spectrum disorder. While a small number of patients that have typical manifestation of anti-NMDAR encephalitis can be combined with lower titer of MOG antibodies in the blood but have no symptoms associated with demyelinating syndrome or changes in brain MRI ${ }^{[8]}$. After this study, there have been some cases of NMDAR antibody encephalitis combined with MOG antibody-related demyelinating disease that are reported. Clinical phenotypes with optic neuritis are relatively common in adults and older children, and others include optic neuromyelitis spectrum disorders and encephalitis ${ }^{[10-13]}$. Recurrent demyelinating episodes occurred in certain patients that require second-line immunotherapy and may leave neurological sequelae, and the most common one is visual impairment ${ }^{[8-12]}$. There are also patients who showed mild NMDAR antibody encephalitis, but brain MRI showed multiple intracranial demyelinating lesions ${ }^{[14]}$.

This study reported 7 child patients with anti-NMDAR antibody encephalitis combined with positive MOG ab and is the largest number of reported cases in children. There are not many related pediatric cases reported so far. Combined with this study and pediatric cases reported, clinical manifestations of the overlap of these two antibodies may have the following 4 characteristics: 1) anti-NMDAR encephalitis and MOG antibody-related demyelinating disease can occur independently, and identification and diagnosis of the two diseases are simple in such case. 2) Anti-NMDAR encephalitis and MOG antibodyrelated demyelinating disease can occur simultaneously. Symptoms of the two diseases exist independently and overlap sometimes. Brain MRI showed cortical, subcortical and subtentorial multifocal lesions. Compared with adults, ADEM was the most common demyelinating event in children. 3) Children with typical NMDAR antibody encephalitis can also be combined with positive MOG antibodies without clinical or imaging demyelination. MOG antibody titer was low and did not last long in these children. 4) 
In this study, no severe cases were reported; the children are all responded well to first-line corticosteroid treatment combined with intravenous immunoglobulin. The relapsed patients were still effective to firstline therapy and had no relapses after prolonged corticosteroid maintenance therapy. Anti-NMDAR encephalitis progresses rapidly, and quite a significant cases require intensive care because of status epilepticus or central hypoventilation ${ }^{[5]}$, while MOG related disease are prone to relapse and leave residual neurological impairment that some of them need to start second-line immunotherapy ${ }^{[15]}$. The overlap of the two antibodies may have a protective effect.

In MOG related disease, recurrence factors include onset age more than 8 year-old, demyelinating type such as optic neuritis and high persistent MOG antibody titer ${ }^{[16]}$. In this study, those with recurrent course may relate to high persistent MOG antibody titer at first onset. Patients with high titer of CSF NMDAR antibody at the first onset may leave neurological sequelae. The overall prognosis was good, 3 cases had neurological dysfunction may related to high NMDAR antibody titer at first onset.

Conclusion: Anti-NMDAR encephalitis combined with positive MOG antibodies is more common in girl around 6 year-old. The most common combination of demyelinating events was ADEM. Children responded well to the first-line treatment of corticosteroid and IVIG. Cases with persistent MOG antibody need longer follow-up due to the risk of recurrence. High NMDAR antibody titer may cause neurological sequelae.

\section{Declarations}

Abbreviations

Ab: Antibody; ADEM: Acute disseminated encephalomyelitis; AQP4: Aquaporin-4; CNS: Central nervous system; CSF: Cerebrospinal fluid; EEG: Electroencephalogram; IVIG: Intravenous immunoglobulin; IVMP: Intravenous methylprednisolone; MOG: Myelin oligodendrocyte glycoprotein; MRI: Magnetic resonance imaging; mRS: modified Rankin Score; MDEM: Multiphasic disseminated encephalomyelitis; NMDAR: NMethyl-D-Aspartate Receptor; NMOSD: Optic myelomyelitis spectrum disorder; Pro: Protein; WBC: White blood cell.

Acknowledgments

The authors thank all the patients and family members who participated in this study.

Funding

The authors have not declared a specific grant for this research from any funding agency in the public, commercial or not-for-profit sectors.

Availability of data and materials 
The datasets generated during and/or analysed during the current study are not publicly available due to local data protection requirements but are available from the corresponding author on reasonable request in an anonymized fashion.

Authors' contributions: Chi Hou: initiated and designed the project and wrote the manuscript; Xiaojing Li and Wenxiong Chen provide critical revision of the manuscript for important intellectual content, study concept and design, study supervision.खWenlin Wu analysed the MRI data. Yang Tian, Yani Zhang and Haixia Zhu were in charge of study concept and design. All other authors collected clinical and paraclinical data, were References involved in patient care, and/or have contributed case reports. All authors were involved in revising the manuscript for intellectual content. All authors read and approved the final draft before submission.

Competing interests

The authors declare that they have no competing interests.

Consent for publication

Participants gave written informed consent for publication of their clinical and paraclinical data.

Ethics approval and consent to participate

The protocol was established, according to the ethical guidelines of the Helsinki Declaration and was approved by the Human Ethics Committee of Guangzhou Women and Children's Medical Center (Approval No: $\$ 2019 ₫ 40701$ ), and Written informed consent was obtained from individual or guardian participants.

Author details

Department of Neurology, Guangzhou Women and Children's Medical Center, Affiliated Hospital of Guangzhou Medical University, 318 Middle Renmin Road, Guangzhou, Guangdong 510120, China.

\section{References}

[1] Dalmau J, Tüzün E, Wu HY, et al. Paraneoplastic anti-N-methyl-D-aspartate receptor encephalitis associated with ovarian teratoma. Ann Neurol. 2007. 61(1): 25-36.

[2] Wynford-Thomas R, Jacob A, Tomassini V. Neurological update: MOG antibody disease. J Neurol. 2019. 266(5): 1280-1286.

[3] Lu J, Samson S, Kass J, Ram N. Acute psychosis in a pregnant patient with Graves' hyperthyroidism and anti-NMDA receptor encephalitis. BMJ Case Rep. 2015. 2015. 
[4] Ran Y, Wang L, Zhang F, Ao R, Dong Z, Yu S. Anti-NMDAR encephalitis followed by seropositive neuromyelitis optica spectrum disorder: A case report and literature review. Clin Neurol Neurosurg. 2017. 155: 75-82.

[5] Staley EM, Jamy R, Phan AQ, Figge DA, Pham HP. N-Methyl-d-aspartate Receptor Antibody Encephalitis: A Concise Review of the Disorder, Diagnosis, and Management. ACS Chem Neurosci. 2019. 10(1): 132-142.

[6] Pruetarat N, Netbaramee W, Pattharathitikul S, Veeravigrom M. Clinical manifestations, treatment outcomes, and prognostic factors of pediatric anti-NMDAR encephalitis in tertiary care hospitals: A multicenter retrospective/prospective cohort study. Brain Dev. 2019. 41(5): 436-442.

[7] Wang L, ZhangBao J, Zhou L, et al. Encephalitis is an important clinical component of myelin oligodendrocyte glycoprotein antibody associated demyelination: a single-center cohort study in Shanghai, China. Eur J Neurol. 2019. 26(1): 168-174.

[8] Titulaer MJ, Höftberger R, lizuka T, et al. Overlapping demyelinating syndromes and anti-N-methylD-aspartate receptor encephalitis. Ann Neurol. 2014. 75(3): 411-28.

[9] Fan S, Xu Y, Ren H, et al. Comparison of myelin oligodendrocyte glycoprotein (MOG)-antibody disease and AQP4-IgG-positive neuromyelitis optica spectrum disorder (NMOSD) when they co-exist with anti-NMDA (N-methyl-D-aspartate) receptor encephalitis. Mult Scler Relat Disord. 2018. 20: 144-152.

[10] Zhou L, ZhangBao J, Li H, et al. Cerebral cortical encephalitis followed by recurrent CNS demyelination in a patient with concomitant anti-MOG and anti-NMDA receptor antibodies. Mult Scler Relat Disord. 2017. 18: 90-92.

[11] Rojc B, Podnar B, Graus F. A case of recurrent MOG antibody positive bilateral optic neuritis and anti-NMDAR encephalitis: Different biological evolution of the two associated antibodies. $J$ Neuroimmunol. 2019. 328: 86-88.

[12] Nagata S, Nishimura Y, Mitsuo K. [A case of anti-myelin oligodendrocyte glycoprotein (MOG) and anti-N-methyl-D-aspartate (NMDA) receptor antibody-positive encephalitis with optic neuritis]. Rinsho Shinkeigaku. 2018. 58(10): 636-641.

[13] Sarigecili E, Cobanogullari MD, Komur M, Okuyaz C. A rare concurrence: Antibodies against Myelin Oligodendrocyte Glycoprotein and N-methyl-d-aspartate receptor in a child. Mult Scler Relat Disord. 2019. 28: 101-103.

[14] Zhou J, Tan W, Tan SE, Hu J, Chen Z, Wang K. An unusual case of anti-MOG CNS demyelination with concomitant mild anti-NMDAR encephalitis. J Neuroimmunol. 2018. 320: 107-110.

[15] Hacohen Y, Wong YY, Lechner C, et al. Disease Course and Treatment Responses in Children With Relapsing Myelin Oligodendrocyte Glycoprotein Antibody-Associated Disease. JAMA Neurol. 2018. 75(4): 
[16] Hennes EM, Baumann M, Schanda K, et al. Prognostic relevance of MOG antibodies in children with an acquired demyelinating syndrome. Neurology. 2017. 89(9): 900-908.

\section{Tables}

Table 1₫Clinical data of the patients

\begin{tabular}{|c|c|c|c|c|c|c|c|}
\hline $\begin{array}{l}\text { Case } \\
\text { NO. }\end{array}$ & Sex & Age(y) & $\begin{array}{l}\text { Symptoms related anti- } \\
\text { NMDAR encephalitis }\end{array}$ & $\begin{array}{l}\text { Sympotoms } \\
\text { demyelination } \\
\text { events }\end{array}$ & $\mathrm{mRS}$ & $\begin{array}{l}\text { Demyelin- } \\
\text { ation type }\end{array}$ & $\begin{array}{l}\text { Relapse } \\
\text { interval } \\
\text { and } \\
\text { phenotype }\end{array}$ \\
\hline 1 & $\overline{\mathrm{M}}$ & 4 & $\begin{array}{l}\text { Personality change, irritability, } \\
\text { mutism, involuntary movement } \\
\text { insomnia }\end{array}$ & प & 3 & प & 0 \\
\hline 2 & $\mathrm{~F}$ & 7 & $\begin{array}{l}\text { irritability } \square \text { involuntary } \\
\text { movement } \rrbracket \text { nightmare } \llbracket \text { mutism } \square \\
\text { seizure }\end{array}$ & $\begin{array}{l}\text { encephalopathy, } \\
\text { paralysis, } \\
\text { facioplegia }\end{array}$ & $\begin{array}{l}4 \\
\text { relapse: } \\
3\end{array}$ & $\mathrm{ADEM}$ & $\begin{array}{l}8 \text { months } \\
\text { anti- } \\
\text { NMDAR } \\
\text { ncephalitis } \\
\text { and ADEM }\end{array}$ \\
\hline 3 & $\mathrm{~F}$ & 6 & $\begin{array}{l}\text { irritability[involuntary } \\
\text { movement』nightmare }] \\
\text { cataphasia }\end{array}$ & $\begin{array}{l}\text { encephalopathy, } \\
\text { paralysis }\end{array}$ & 4 & $\mathrm{ADEM}$ & 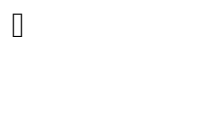 \\
\hline 4 & $\mathrm{~F}$ & 5 & $\begin{array}{l}\text { Personality change } \llbracket \text { reversed } \\
\text { sleep circadian rhythm, unclear } \\
\text { pronounce } \llbracket \text { seizure }\end{array}$ & encephalopathy & 3 & $\mathrm{ADEM}$ & ? \\
\hline 5 & $\mathrm{~F}$ & 6 & $\begin{array}{l}\text { 1-year after } \square \text { irritability } \square \\
\text { insomnia } \llbracket \text { seizure }\end{array}$ & $\begin{array}{l}\text { First onset } \square \\
\text { encephalopathy, } \\
\text { paralysis, } \\
\text { facioplegia }\end{array}$ & $\begin{array}{l}4 \\
\text { 1-year } \\
\text { after : } 3\end{array}$ & $\mathrm{ADEM}$ & प \\
\hline 6 & $\mathrm{~F}$ & 8 & $\begin{array}{l}\text { Personality change } \square \text { irritability, } \\
\text { mutism insomnia } \square \text { involuntary } \\
\text { movement }\end{array}$ & ] & 3 & - & प \\
\hline 7 & M & 6 & irritability $\square$ mutism, seizure & $\begin{array}{l}\text { encephalopathy, } \\
\text { paralysis }\end{array}$ & $\begin{array}{l}4, \\
\text { relapse: } \\
3\end{array}$ & $\mathrm{ADEM}$ & $\begin{array}{l}6 \text { months } \\
\text { ADEM }\end{array}$ \\
\hline
\end{tabular}

ADEM: acute disseminated encephalomyelitis; NMDAR: Nmethyl- D-aspartate receptor; mRS: modified Rankin score 


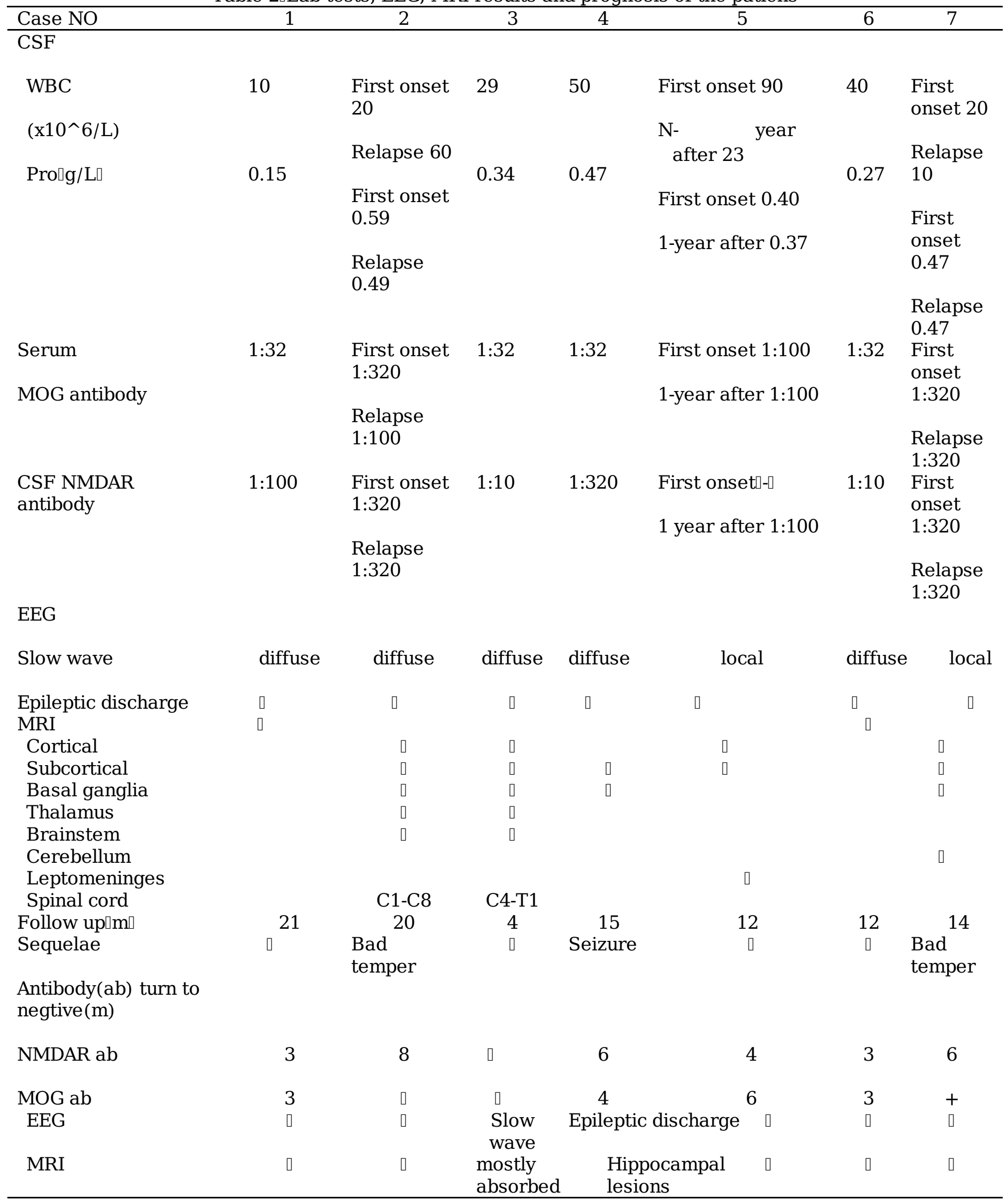


CSF: cerebrospinal fluid; WBC: white blood cell; Pro: protein; NMDAR: Nmethyl- D-aspartate receptor; MOG: myelin oligodendrocyte glycoprotein; ab: antibody; EEG: electroencephalogram; MRI: magnetic resonance imaging

\section{Figures}

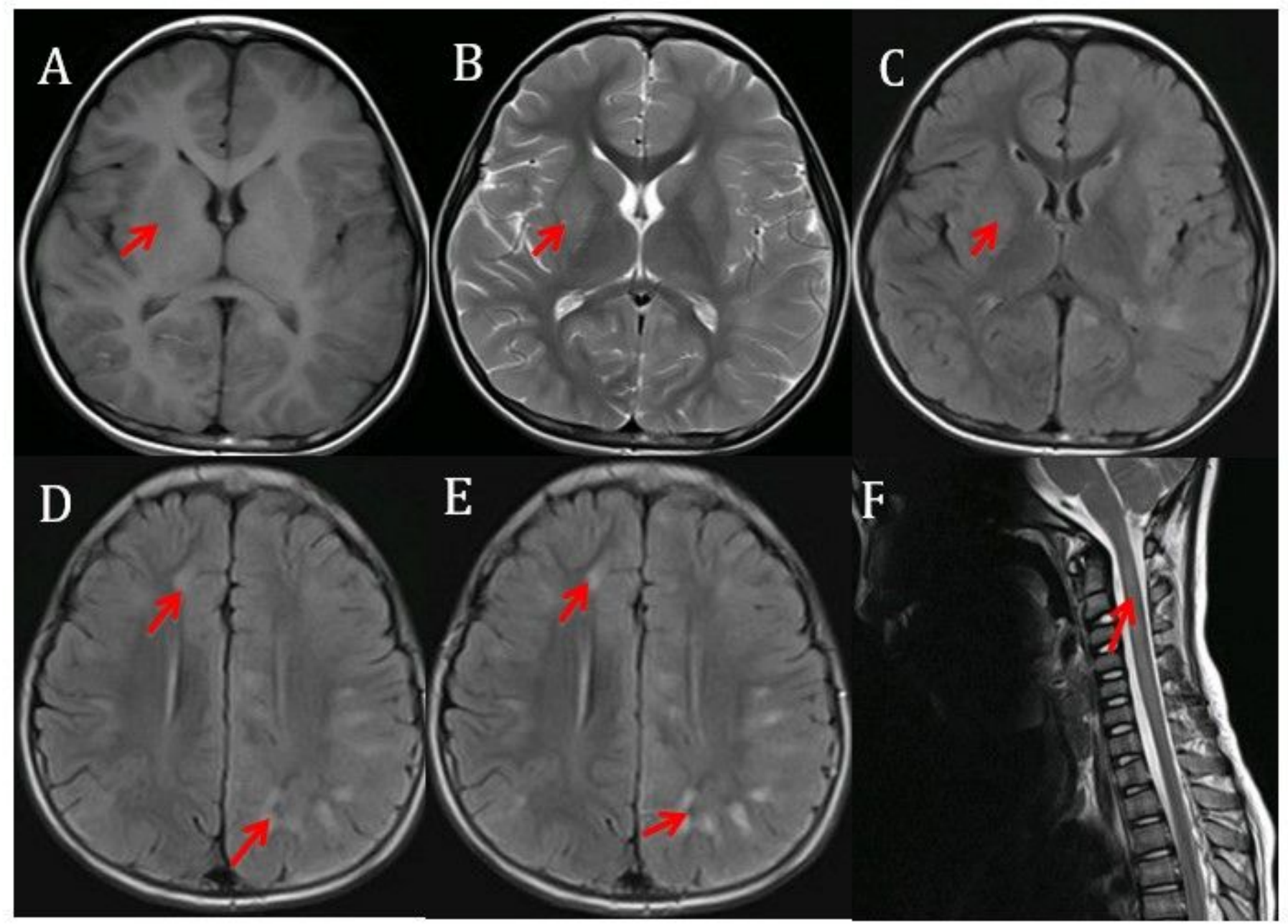

\section{Figure 1}

: 3T MRI of a patients with NMDAR encephalitis combined with MOG antibody related ADEM: A, B, C, D: patchy abnormal signal shadow in bilateral basal ganglia region, $A$ : lesions show slightly low signal on T1WI, B and C: lesions show high signal on T2WI and T2WI-FLAIR, D: Subcortical white matter lesions in bilateral frontal and parietal lobe; E: lesions show mild enhancement on T2WI-FLAIR enhanced scan; F: T2WI cervical spinal cord is slightly swollen, and a few patchy high signal shadows 